M. Kato.

Nagoya Math. J

Vol. 44 (1971). 119-132

\title{
STABLE MANIFOLDS OF A MAP AND A FLOW FOR A COMPACT MANIFOLD
}

\author{
MASAHARU KATO
}

\section{$\S 0$. Introduction}

The purpose of this paper is to generalize the notion of the stable manifolds in Smale [5] and [6], in which the stable manifolds of flows or diffeomorphisms for a singular point or a closed orbit are defined in certain conditions. This generalization is concerned with Fenichel [1]. He considers the stable manifolds of flows and diffeomorphisms for a torus. Here, we consider the case of a compact manifold. But our argument does not exactly imply Fenichel's result.

It is interesting to investigate the conditions for the existence of stable manifolds of flows or diffeomorphisms. If the stable manifolds exist, then we can see to some extent the state of the orbits of flows or diffeomorphisms near the stable manifolds.

In $\S 1$, we prove Theorem 1 by the method of successive approximations and we obtain a local stable manifold of a map for a compact manifold as a graph of the solution map. In Corollary of Theorem 1, we study the state of the orbits of a map. In $\$ 2$, we construct a local stable manifold of a flow by using the result of $\S 1$ and we study the state of the orbits of the flow.

The author wishes to express his gratitude to Professor Shiraiwa who gave him many valuable advices very kindly.

\section{§1. The stable manifold of a map.}

First, we shall explain the notations.

Let $T$ be a compact $C^{l}$-manifold $(1 \leq l<\infty)$ and $E_{i}$ be a $k_{i}$ dimensional Euclidean space for $i=1,2$. We denote by $L_{i}$ a $k_{i} \times k_{i}$ non-singular matrix for $i=1$, 2. Define the norm of a vector $z=\left(z_{1}, \cdots, z_{k_{i}}\right) \in E_{i}$ (resp. a matrix $\left.L_{i}\right)$ by $\|z\|=\max \left(\left|z_{1}\right|, \cdots,\left|z_{k_{i}}\right|\right)$ (resp. $\left.\left\|L_{i}\right\|=\sup _{\|z\|=1}\left\|L_{i} z\right\|\right)$. We suppose

Received October, 301970 
that the norms of $L_{1}$ and $L_{2}^{-1}$ are less than one. If we put $\left\|L_{1}\right\|=a$ and $\left\|L_{2}^{-1}\right\|=\frac{1}{b}$, then $a<1<b$. Put $B_{i}\left(r_{0}\right)=\left\{z \in E_{i} \mid\|z\| \leq r_{0}\right\}$ for a positive constant $r_{0}$. Let $\xi: T \times B_{1}\left(r_{0}\right) \times B_{2}\left(r_{0}\right) \rightarrow T$ be of class $C^{l}$ and let $\varphi_{i}: T \times B_{1}\left(r_{0}\right) \times B_{2}\left(r_{0}\right)$ $\rightarrow E_{i}$ be of class $C^{l}$ for $i=1$, 2. Define $F: T \times B_{1}\left(r_{0}\right) \times B_{2}\left(r_{0}\right) \rightarrow T \times E_{1} \times E_{2}$ by $F(\tau, x, y)=\left(\xi(\tau, x, y), L_{1} x+\varphi_{1}(\tau, x, y), L_{2} y+\varphi_{2}(\tau, x, y)\right)$.

For defining the norm of $d \xi(\tau, x, y)$ and $\left\|\tau-\tau^{\prime}\right\|$ for sufficiently close $\tau$, $\tau^{\prime}$ of $T$, we shall give the following remarks.

In general, let $M$ and $N$ be two compact $C^{1}$-manifolds and $h: M \rightarrow N$ be a $C^{1}$-map. Let $\left\{V_{j}\right\}_{j=1}^{n}$ be a finite open covering of $N$ by ${ }^{*}$ nice coordinate neighborhoods $V_{j}$. Next, let $\left\{U_{i}\right\}_{i=1}^{m}$ be a finite open covering of $M$ by ${ }^{*}$ nice coordinate neighborhoods $U_{i}$ such that for each $i h\left(U_{i}\right)$ is contained in a suitable $V_{j}$. Let $\left\{h_{i}\right\}_{i=1}^{m}$ (resp. $\quad\left\{h_{j}^{\prime}\right\}_{j=1}^{n}$ ) be a coordinate system associated with $\left\{U_{i}\right\}_{i=1}^{n}$ (resp. $\left\{V_{j}\right\}_{j=1}^{n}$ ). We define the norm of $d h$ by $\|d h\|=\max _{x \in U_{i^{\prime}}}$ $\left\|d h_{j}^{\prime} h h_{i}^{-1}(x)\right\|$ for each $(i, j)$ such that $h\left(U_{i}\right) \subset V_{j}$. When $m$ and $m^{\prime}$ are sufficiently close in $M$, we put $\left\|m-m^{\prime}\right\|=\max \left\|h_{i}(m)-h_{i}\left(m^{\prime}\right)\right\|$ for each $i$ such that $m, m^{\prime} \in U_{i}$.

By the first remark, we can define the norm of $d \xi(\tau, x, y)$ and we put $c=\max \left\{\|d \xi(\tau, x, y)\| \mid \tau \in T, x \in B_{1}\left(r_{0}\right), y \in B_{2}\left(r_{0}\right)\right\}$. Also, we can use the mean value theorem with respect to the first variable by the second remark.

Let $f: M_{1} \times M_{2} \times M_{3} \rightarrow N$ be a $C^{l}$-map. We denote by $d_{i} f$ the partial derivative of $f$ with respect to the $i$-th variable for $i=1,2,3$ and by $d_{(2,3)} f$ the partial derivative of $f$ with respect to the second and third variables.

Theorem 1. Suppose that

$$
\varphi_{i}(\tau, 0,0)=0, d_{(2,3)} \varphi_{i}(\tau, 0,0)=0, i=1,2,
$$

for any $\tau \in T$ and $c, c^{2}, \cdots, c^{l^{\prime}}<b\left(1 \leq l^{\prime} \leq l\right)$, then there exist a positive number $\delta$ and $a C^{\prime \prime}$ map $g: T \times B_{1}(\delta) \rightarrow E_{2}$ such that

$$
g(\tau, 0)=0, d_{2} g(\tau, 0)=0 \text { for any } \tau \in T,
$$

Corollary. For a fixed $\left(\tau_{0}, x_{0}, y_{0}\right) \in T \times B_{1}\left(r_{0}\right) \times B_{2}\left(r_{0}\right)$, we put $\left(\tau_{1}, x_{1}, y_{1}\right)=$ $F\left(\tau_{0}, x_{0}, y_{0}\right)$. If $\left(\tau_{m}, x_{m}, y_{m}\right) \in T \times B_{1}\left(r_{0}\right) \times B_{2}\left(r_{0}\right)$, then we define $\left(\tau_{m+1}, x_{m+1}, y_{m+1}\right)$ $=F\left(\tau_{m}, x_{m}, y_{m}\right)$.

*) Let $\left\{U_{i}\right\}_{i=1}^{n}$ be a finite open covering of a compact manifold $M$ by coordinate neighborhoods and $U_{i}^{\prime}\left(\subset U_{i}\right)$ be compact and $\bigcup_{i=1}^{n} \operatorname{Int} U_{i}^{\prime}=M$. We call these $\left\{\left(U_{i}, U_{i}^{\prime}\right)\right\}_{i=1}^{n}$ nice coordinate neighborhoods. 
If $y_{0}=g\left(\tau_{0}, x_{0}\right)$, then $y_{m}=g\left(\tau_{m}, x_{m}\right)$ for any $m$ and $\left\|\left(x_{m}, y_{m}\right)\right\|=O\left(\left(a+2 \theta_{1}\right)^{m}\right)$ as $m \rightarrow \infty$, where $\theta_{1}$ is a sufficiently small positive number.

If $y_{0} \neq g\left(\tau_{0}, x_{0}\right)$, then $y_{m} \neq g\left(\tau_{m}, x_{m}\right)$ as far as $\left(\tau_{m}, x_{m}, y_{m}\right)$ is defined. Actually, there exists a constant $d>1$ such that $\left\|y_{m}-g\left(\tau_{m}, x_{m}\right)\right\| \geq d^{m}\left\|y_{0}-g\left(\tau_{0}, x_{0}\right)\right\|$ and also $\left\|\left(x_{m}, y_{m}\right)\right\| \geq \frac{1}{2} d^{m}\left\|y_{0}-g\left(\tau_{0}, x_{0}\right)\right\|$ as far as $\left(\tau_{m}, x_{m}, y_{m}\right)$ is defined.

Let $V=\{(\tau, x, g(\tau, x)) \mid \tau \in T,\|x\|<\delta\}$, then $V$ is a $C^{\prime \prime}$-manifold and (1.3) implies $F(V) \subset V$. By Corollary of Theorem $1, V$ consists of the point $(\tau, x, y)$ such that $\lim _{n \rightarrow \infty} F^{n}(\tau, x, y) \in T \times 0 \times 0$. We call $V$ the local stable manifold of a map $F$ for a compact manifold $T$.

If $F: T \times B_{1}\left(r_{0}\right) \times B_{2}\left(r_{0}\right) \rightarrow T \times E_{1} \times E_{2}$ is an imbedding, $\bigcup_{n=0}^{\infty} F^{-n}(V)$ is a $C^{l^{\prime}}-$ manifold and is called the stable manifold of a map $F$ for a compact manifold $T$. We can define the unstable manifold by a similar method.

Proof of Theorem 1. Let $\theta_{1}$ be a sufficiently small positive number, which will be determined later so that the following arguments hold. (1.1) implies that for any $\theta_{1}>0$ there exists a positive number $r\left(\leq r_{0}\right)$ such that

$$
\left\|d \varphi_{1}\right\|,\left\|d \varphi_{2}\right\| \leq \theta_{1} \text { for } \tau \in T \text { and }\|x\| \leq r .
$$

Since $\varphi_{1}, \varphi_{2}$ and $\xi$ are $C^{l}$-maps, there exists a positive number $\theta_{m}$ $(m=2,3, \cdots, l)$ such that

$$
\left\|d^{m} \varphi_{1}\right\|,\left\|d^{m} \varphi_{2}\right\|,\left\|d^{m} \xi\right\| \leq \theta_{m} \text { for } \tau \in T,\|x\| \leq r \text { and }\|y\| \leq r .
$$

Denote by $\Gamma$ the set of the map $f$ with the following properties:

$$
y=f(\tau, x): T \times B_{1}(\delta) \rightarrow E_{2} \text { is of class } C^{l^{\prime}},
$$

where $\delta=\frac{\theta_{1}}{1-a-\theta_{1}}-r$. Let $\theta_{1}<\frac{1-a}{2}$. Then $0<\delta \leq r$.

$$
\begin{gathered}
f(\tau, 0)=0, d_{2} f(\tau, 0)=0 \text { for any } \tau \in T . \\
\text { If } \tau \in T \text { and }\|x\| \leq \delta, \text { then }
\end{gathered}
$$

(a) $\|f(\tau, x)\| \leq r$

(b) $\left\|L_{1} x+\varphi_{1}(\tau, x, f(\tau, x))\right\| \leq \delta$,

(c) $\left\|L_{2}^{-1}\left[f\left(\xi(\tau, x, f(\tau, x)), L_{1} x+\varphi_{1}(\tau, x, f(\tau, x))\right)-\varphi_{2}(\tau, x, f(\tau, x))\right]\right\| \leq r$.

$$
\|d f(\tau, x)\| \leq \sigma_{1} \text { for any } \tau \in T \text { and }\|x\| \leq \delta,
$$

where $\sigma_{1}=\frac{\theta_{1}}{b-\max (c, 1)}$. Let $\theta_{1}<b-\max (c, 1)$. Then $0<\sigma_{1}<1$.

Define a map $\Phi: \Gamma \rightarrow \Gamma$ by $\Phi(f)=\psi$, where $\psi(\tau, x)=L_{2}^{-1}[f(\xi(\tau, x, f(\tau, x))$, $\left.\left.L_{1} x+\varphi_{1}(\tau, x, f(\tau, x))\right)-\varphi_{2}(\tau, x, f(\tau, x))\right]$. We shall show $\phi \in \Gamma$. It is trivial 
that $\phi$ satisfies $(1.6), \psi(\tau, 0)=0$ and (a) of (1.8). First, we shall show $d_{2}(\tau, 0)$ $=0$. Since

$$
\begin{aligned}
d_{2} \psi(\tau, x)= & L_{2}^{-1}\left[d _ { 1 } f ( \xi ( \tau , x , f ( \tau , x ) ) , L _ { 1 } x + \varphi _ { 1 } ( \tau , x , f ( \tau , x ) ) ) \cdot \left\{d_{2} \xi(\tau, x, f(\tau, x))\right.\right. \\
& \left.+d_{3} \xi(\tau, x, f(\tau, x)) \cdot d_{2} f(\tau, x)\right\}+d_{2} f\left(\xi(\tau, x, f(\tau, x)), L_{1} x\right. \\
& \left.+\varphi_{1}(\tau, x, f(\tau, x))\right) \cdot\left\{L_{1}+d_{2} \varphi_{1}(\tau, x, f(\tau, x))\right. \\
& \left.+d_{3} \varphi_{1}(\tau, x, f(\tau, x)) \cdot d_{2} f(\tau, x)\right\}-d_{2} \varphi_{2}(\tau, x, f(\tau, x)) \\
& \left.-d_{3} \varphi_{2}(\tau, x, f(\tau, x)) \cdot d_{2} f(\tau, x)\right],
\end{aligned}
$$

$d_{2} \psi(\tau, 0)=L_{2}^{-1}\left[d_{1} f((\tau, 0,0), 0) \cdot d_{2} \xi(\tau, 0,0)\right]$ by $(1.1)$ and (1.7).

Since $f(\tau, 0)=0, d_{1} f(\tau, 0)=0$. Therefore, $d_{2} \psi(\tau, 0)=0$.

Next, we shall prove (b) of (1.8). Let $\tau \in T$ and $\|x\| \leq \delta$. Then

$$
\begin{aligned}
& \left\|L_{1} x+\varphi_{1}(\tau, x, \psi(\tau, x))\right\| \\
& \leq a \delta+\left\|\varphi_{1}(\tau, x, \psi(\tau, x))-\varphi_{1}(\tau, x, 0)\right\|+\left\|\varphi_{1}(\tau, x, 0)-\varphi_{1}(\tau, 0,0)\right\| \\
& \leq a \delta+\theta_{1}\|\psi(\tau, x)\|+\theta_{1}\|x\| \quad \text { (mean value theorem and (1.4)) } \\
& \left.\leq a \delta+\theta_{1} r+\theta_{1} \delta \quad \text { ((a) of (1.8) for } \psi\right) \\
& \leq \delta
\end{aligned}
$$

Now, we shall prove (c) of (1.8). Let $\tau \in T$ and $\|x\| \leq \delta$. Then

$$
\begin{aligned}
& \left\|L_{2}^{-1}\left[\psi\left(\xi(\tau, x, \psi(\tau, x)), L_{1} x+\varphi_{1}(\tau, x, \phi(\tau, x))\right)-\varphi_{2}(\tau, x, \psi(\tau, x))\right]\right\| \\
\leq & \frac{1}{b}\left[\left\|\psi\left(\xi(\tau, x, \psi(\tau, x)), L_{1} x+\varphi_{1}(\tau, x, \psi(\tau, x))\right)\right\|+\left\|\varphi_{2}(\tau, x, \phi(\tau, x))\right\|\right] \\
\leq & \frac{1}{b}\left(r+\theta_{1} r+\theta_{1} \delta\right) \\
\leq & \frac{1}{b}\left(1+2 \theta_{1}\right) r .
\end{aligned}
$$

Let $\theta_{1}<\frac{b-1}{2}$. Then $\frac{1}{b}\left(1+2 \theta_{1}\right) r \leq r$.

Finally, we shall show (1.9). Since

$$
\begin{aligned}
d \psi(\tau, x)= & L_{2}^{-1}\left[d f\left(\xi(\tau, x, f(\tau, x)), L_{1} x+\varphi_{1}(\tau, x, f(\tau, x))\right) \cdot(d \xi(\tau, x, f(\tau, x)) \cdot\right. \\
& \left.(1,1, d f(\tau, x)), L_{1}+d \varphi_{1}(\tau, x, f(\tau, x)) \cdot(1,1, d f(\tau, x))\right) \\
- & \left.d \varphi_{2}(\tau, x, f(\tau, x)) \cdot(1,1, d f(\tau, x))\right], \\
\|d \psi(\tau, x)\| \leq & \frac{1}{b}\left(\sigma_{1} \max \left(c, a+\theta_{1}\right)+\theta_{1}\right) .
\end{aligned}
$$

Let $a+\theta_{1}<1$. Then $\frac{1}{b}\left(\sigma_{1} \max \left(c, a+\theta_{1}\right)+\theta_{1}\right) \leq \frac{1}{b}\left(\sigma_{1} \max (c, 1)+\theta_{1}\right) . \quad$ By 
the definition of $\sigma_{1}$, it is easy to see that the above right term equals to $\sigma_{1}$.

Thus, we have $\phi \in \Gamma$ and the map $\Phi$ is well defined.

Define $g_{0}(\tau, x) \equiv 0$ for any $\tau \in T$ and $\|x\| \leq \delta$, then it is easy to see $g_{0} \in \Gamma$. Put $\Phi\left(g_{0}\right)=g_{1}, \Phi\left(g_{1}\right)=g_{2}, \cdots$, then $g_{1}, g_{2}, \cdots \in \Gamma$.

It will be verified by induction that there exist a positive number $k(<1)$ and $M$ such that

$$
\left\|g_{m}(\tau, x)-g_{m-1}(\tau, x)\right\| \leq M k^{m} \text { for } \tau \in T \text { and }\|x\| \leq \delta .
$$

For $m=1$,

$$
\left\|g_{1}(\tau, x)-g_{0}(\tau, x)\right\| \leq\left\|L_{2}^{-1}\left(-\varphi_{2}(\tau, x, 0)\right)\right\| \leq \frac{\theta_{1}}{b} \delta .
$$

Put $M k=\frac{\theta_{1}}{b} \delta$, then (1.10) holds for $m=1$. Assume that (1.10) holds if $m$ is replaced by $m-1$. Then

$$
\begin{aligned}
& \left\|g_{m}(\tau, x)-g_{m-1}(\tau, x)\right\| \\
\leq & \frac{1}{b}\left[\| g_{m-1}\left(\xi\left(\tau, x, g_{m-1}(\tau, x)\right), L_{1} x+\varphi_{1}\left(\tau, x, g_{m-1}(\tau, x)\right)\right)\right. \\
& \quad-g_{m-2}\left(\xi\left(\tau, x, g_{m-1}(\tau, x)\right), L_{1} x+\varphi_{1}\left(\tau, x, g_{m-1}(\tau, x)\right)\right) \| \\
+ & \| g_{m-2}\left(\xi\left(\tau, x, g_{m-1}(\tau, x)\right), L_{1} x+\varphi_{1}\left(\tau, x, g_{m-1}(\tau, x)\right)\right) \\
& \quad-g_{m-2}\left(\xi\left(\tau, x, g_{m-1}(\tau, x)\right), L_{1} x+\varphi_{1}\left(\tau, x, g_{m-2}(\tau, x)\right)\right) \| \\
+ & \| g_{m-2}\left(\xi\left(\tau, x, g_{m-1}(\tau, x)\right), L_{1} x+\varphi_{1}\left(\tau, x, g_{m-2}(\tau, x)\right)\right) \\
& \quad-g_{m-2}\left(\xi\left(\tau, x, g_{m-2}(\tau, x)\right), L_{1} x+\varphi_{1}\left(\tau, x, g_{m-2}(\tau, x)\right)\right) \| \\
+ & \left.\left\|\varphi_{2}\left(\tau, x, g_{m-1}(\tau, x)\right)-\varphi_{2}\left(\tau, x, g_{m-2}(\tau, x)\right)\right\|\right] .
\end{aligned}
$$

By the assumption of induction and the mean value theorem,

$$
\begin{aligned}
& \left\|g_{m}(\tau, x)-g_{m-1}(\tau, x)\right\| \\
\leq & \frac{1}{b}\left(M k^{m-1}+\sigma_{1} \theta_{1} M k^{m-1}+\sigma_{1} c M k^{m-1}+\theta_{1} M k^{m-1}\right) \\
\leq & \frac{1}{b} M k^{m-1}\left(1+\sigma_{1} \theta_{1}+\sigma_{1} c+\theta_{1}\right) .
\end{aligned}
$$

We put $k=\frac{1}{b}\left(1+\sigma_{1} \theta_{1}+\sigma_{1} c+\theta_{1}\right)$. For a sufficiently small $\theta_{1}$ the following inequality holds: $\sigma_{1} \theta_{1}+\sigma_{1} c+\theta_{1}<b-1\left(\right.$ Note $\left.\sigma_{1}=\frac{\theta_{1}}{b-\max (c, 1)}\right)$. For such a $\theta_{1}$ we have $0<k<1$ and $\left\|g_{m}(\tau, x)-g_{m-1}(\tau, x)\right\| \leq M k^{m}$. Therefore, $g(\tau, x)$ $=\lim _{m \rightarrow \infty} g_{m}(\tau, x)$ exists uniformly for $\tau \in T$ and $\|x\| \leq \delta$. It is trivial that $g$ is continuous and $g$ satisfies $g(\tau, 0)=0$ and (1.3). 
Next, it will be proved that the solution map $g$ is of class $C^{1}$. For any map $f=f(u)$ (resp. $f=f(v, w)$ ), let $\Delta f=f(u+\Delta u)-f(u)$ (resp. $\Delta f=$ $f(v+\Delta v, w+\Delta w)-f(v, w))$. Put $v_{1}(\varepsilon)=\sup \left(\|\Delta d \xi\|,\left\|\Delta d \varphi_{1}\right\|,\left\|\Delta d \varphi_{2}\right\|\right)$ for $\|\Delta \tau\|$, $\|\Delta x\|,\|\Delta y\| \leq \varepsilon$. We shall show that

$$
\|\Delta d f\| \leq u_{1}(\varepsilon) \text { implies }\|\Delta d \psi\| \leq u_{1}(\varepsilon),
$$

where $f \in \Gamma, \Phi(f)=\psi$ and $u_{1}(\varepsilon)=K_{1} v_{1}(\varepsilon)$ and $K_{1}=\frac{1+\sigma_{1}}{b-\max (c, 1)-\sigma_{1} c-\theta_{1}}$. For a sufficiently small $\theta_{1}, b-\max (c, 1)>\sigma_{1} c+\theta_{1}\left(\right.$ Note $\left.\sigma_{1}=\frac{\theta_{1}}{b-\max (c, 1)}\right)$. For such a $\theta_{1}$ we have $K_{1}>0$. Assume $\|\Delta d f\| \leq u_{1}(\varepsilon)$. By using the analogue of $\Delta\left[f_{1}(u) f_{2}(u)\right]=f_{1}(u+\Delta u) \Delta f_{2}+\left(\Delta f_{1}\right) f_{2}(u)$, we have the following equation:

$$
\begin{aligned}
\Delta d \psi= & L_{2}^{-1}\left[\Delta d f\left(d \xi(1,1, d f), L_{1}+d \varphi_{1}(1,1, d f)\right)+d f(\Delta d \xi(1,1, d f)\right. \\
& \left.+d \xi(0,0, \Delta d f), \Delta d \varphi_{1}(1,1, d f)+d \varphi_{1}(0,0, \Delta d f)\right)-\Delta d \varphi_{2}(1,1, d f) \\
& \left.-d \varphi_{2}(0,0, \Delta d f)\right] .
\end{aligned}
$$

Then $\|\Delta d \psi\| \leq \frac{1}{b}\left(u_{1}(\varepsilon) \max \left(c, a+\theta_{1}\right)+\sigma_{1} \max \left(v_{1}(\varepsilon)+c u_{1}(\varepsilon), v_{1}(\varepsilon)+\theta_{1} u_{1}(\varepsilon)\right)\right.$

$$
\begin{aligned}
&\left.+v_{1}(\varepsilon)+\theta_{1} u_{1}(\varepsilon)\right) \\
& \leq \frac{1}{b}\left(u_{1}(\varepsilon) \max \left(c, a+\theta_{1}\right)+\sigma_{1} v_{1}(\varepsilon)+\sigma_{1} c u_{1}(\varepsilon)+v_{1}(\varepsilon)+\theta_{1} u_{1}(\varepsilon)\right) \\
& \quad \quad\left(\text { let } \theta_{1}<c\right)
\end{aligned}
$$

By the definition of $u_{1}(\varepsilon)$, it is easy to see that the above term equals to $u_{1}(\varepsilon)$. Therefore, $\|\Delta d \psi\| \leq u_{1}(\varepsilon)$. Since $g_{0} \in \Gamma$ and $\left\|\Delta d g_{0}\right\| \leq u_{1}(\varepsilon)$, we have $\left\|\Delta d g_{m}\right\| \leq u_{1}(\varepsilon)$ for $m=1,2, \cdots$, that is, $\left\{d g_{0}, d g_{1}, \cdots\right\}$ is equi-continuous. Since $g_{m} \in \Gamma,\left\{d g_{0}, d g_{1}, \cdots\right\}$ is uniformly bounded. Therefore, $g$ is of class $C^{1}$ and $d_{2} g(\tau, 0)=0$ since $d_{2} g_{m}(\tau, 0)=0$.

Next, we shall show that the solution map $g$ is of class $C^{\prime \prime}$. Define $\zeta_{j}(\tau, x)=\varphi_{j}(\tau, x, f(\tau, x))$ for $j=1,2,3$, where $\zeta_{3}(\tau, x)=\zeta(\tau, x, f(\tau, x))$. By induction, it is easy to see that

$$
d^{m} \zeta_{j}=d^{m} \varphi_{j}(1,1, d f)^{m}+P_{1}+d \varphi_{j}\left(0,0, d^{m} f\right)
$$

for $m=2,3, \cdots, l^{\prime}$, where $P_{1}$ is a polynomial of $d^{k} \varphi_{j},(1,1, d f)^{k}$ and $d^{k} f$ for $k \leq m-1$. Also, put $\eta(\tau, x)=f\left(\zeta_{3}(\tau, x), L_{1} x+\zeta_{1}(\tau, x)\right)$. By induction, it is easy to see that 


$$
d^{m} \eta=d^{m} f\left(d \zeta_{3}, L_{1}+d \zeta_{1}\right)^{m}+P_{2}+d f\left(d^{m} \zeta_{3}, d^{m} \zeta_{1}\right)
$$

for $m=2,3, \cdots, l^{\prime}$, where $P_{2}$ is a polynomial of $d^{k} f,\left(d \zeta_{3}, L_{1}+d \zeta_{1}\right)^{k}$ and $\left(d^{k} \zeta_{3}, d^{j} \zeta_{1}\right)$ for $j, k \leq m-1$. Since $\phi=L_{2}^{-1}\left(\eta-\zeta_{2}\right)$,

$$
d^{m} \psi=L_{2}^{-1}\left(d^{m} \eta-d^{m} \zeta_{2}\right) \text { for } m=2,3, \cdots, l^{\prime} .
$$

We shall show by induction that there exists a positive number $\sigma_{m}(m=$ $\left.2,3, \cdots, l^{\prime}\right)$ such that

$$
\left\|d^{m} f\right\| \leq \sigma_{m} \text { implies }\left\|d^{m} \psi\right\| \leq \sigma_{m}
$$

where $f \in \Gamma$ and $\Phi(f)=\psi$. For $m=2$, put $\sigma_{2}=\frac{1+\sigma_{1}}{b-[\max (c, 1)]^{2}-\sigma_{1} c-\theta_{1}} \theta_{2}$. Since $c^{2}<b, \sigma_{2}>0$ for a small $\theta_{1}$ such that $b-[\max (c, 1)]^{2}>\sigma_{1} c+\theta_{1}$ (Note $\left.\sigma_{1}=\frac{\theta_{1}}{b-\max (c, 1)}\right)$. Assume $\left\|d^{2} f\right\| \leq \sigma_{2}$. By a simple calculation, we have

$$
\begin{gathered}
d^{2} \eta=d^{2} f\left(d \zeta_{3}, L_{1}+d \zeta_{1}\right)^{2}+d f\left(d^{2} \zeta_{3}, d^{2} \zeta_{1}\right), \\
d^{2} \zeta_{2}=d^{2} \varphi_{2}(1,1, d f)^{2}+d \varphi_{2}\left(0,0, d^{2} f\right) .
\end{gathered}
$$

By (1.14), (1.16) and (1.17),

$$
\begin{aligned}
\left\|d^{2} \psi\right\| & \leq \frac{1}{b}\left(\sigma_{2}\left[\max \left(c, a+\theta_{1}\right)\right]^{2}+\sigma_{1} \max \left(\theta_{2}+c \sigma_{2}, \theta_{2}+\sigma_{1} \theta_{2}\right)+\theta_{2}+\theta_{1} \sigma_{2}\right) \\
& \leq \frac{1}{b}\left(\sigma_{2}[\max (c, 1)]^{2}+\sigma_{1} \theta_{2}+\sigma_{1} c \sigma_{2}+\theta_{2}+\theta_{1} \sigma_{2}\right) \\
& =\frac{1}{b}\left(\sigma_{2}\left([\max (c, 1)]^{2}+\sigma_{1} c+\theta_{1}\right)+\theta_{2}\left(1+\sigma_{1}\right)\right) .
\end{aligned}
$$

By the definition of $\sigma_{2}$, it is easy to see that the above term equals to $\sigma_{2}$. Thus, we have $\left\|d^{2} \psi\right\| \leq \sigma_{2}$. Next, assume that (1.15) holds for $m=2,3, \cdots$, $p-1$. Therefore, we can assume that $\sigma_{1}, \sigma_{2}, \cdots, \sigma_{p-1}$ are defined. By (1.12), (1.13) and (1.14), we have the following inequality:

$$
\begin{aligned}
\left\|d^{p} \psi\right\| \leq & \frac{1}{b}\left(\sigma_{p}\left[\max \left(c, a+\theta_{1}\right)\right]^{p}+\left\|P_{2}\right\|\right. \\
& \left.+\sigma_{1} \max \left(\theta_{p}+\left\|P_{1}\right\|+c \sigma_{p}, \theta_{p}+\left\|P_{1}^{\prime}\right\|+\theta_{1} \sigma_{p}\right)+\theta_{p}+\left\|P_{3}\right\|+\theta_{1} \sigma_{p}\right) \\
\leq & \left.\frac{1}{b}\left(\sigma_{p}[\max (c, 1)]^{p}+\sigma_{1} c+\theta_{1}\right)+\alpha\right),
\end{aligned}
$$

where $\alpha$ is a positive constant. Put $\sigma_{p}=\frac{\alpha}{b-[\max (c, 1)]^{p}-\sigma_{1} c-\theta_{1}}$, then the above term equals to $\sigma_{p}$. Since $c^{p}<b, \sigma_{p}>0$ for a small $\theta_{1}$ such that $b-[\max (c, 1)]^{p}>\sigma_{1} c+\theta_{1}\left(\right.$ Note $\left.\sigma_{1}=\frac{\theta_{1}}{b-\max (c, 1)}\right)$. Thus, we prove (1.15). 
Since $g_{0} \in \Gamma$ and $\left\|d^{m} g_{0}\right\| \leq \sigma_{m}$ for $m=2, \cdots, l^{\prime}$, we have $\left\|d^{m} g_{k}\right\| \leq \sigma_{m}$ for $k=$ $1,2, \cdots$ That is, $\left\{d^{m} g_{0}, d^{m} g_{1}, \cdots\right\}$ is uniformly bounded. Next, put $v_{m}(\varepsilon)=\sup _{\|\Delta \tau\|,\|\Delta x\|,\|\Delta y\| \leq \varepsilon}\left(\left\|\Delta d^{m} \xi\right\|,\left\|\Delta d^{m} \varphi_{1}\right\|,\left\|\Delta d^{m} \varphi_{2}\right\|\right)$ for $m=2,3, \cdots, l^{\prime}$. By induction, it is easy to see that

$$
\begin{gathered}
\Delta d^{m} \zeta_{j}=\Delta d^{m} \varphi_{j}(1,1, d f)^{m}+P+d \varphi_{j}\left(0,0, \Delta d^{m} f\right), \\
\Delta d^{m} \eta=\Delta d^{m} f\left(d \zeta_{3}, L_{1}+d \zeta_{1}\right)^{m}+Q+d f\left(\Delta d^{m} \zeta_{3}, \Delta d^{m} \zeta_{1}\right),
\end{gathered}
$$

where $P$ and $Q$ are suitable polynomials. Also,

$$
\Delta d^{m} \psi=L_{2}^{-1}\left(\Delta d^{m} \eta-\Delta d^{m} \zeta_{2}\right) .
$$

Replacing (1.12), (1.13) and (1.14) by (1.18), (1.19) and (1.20) respectively, we can prove similary as above that there exists a positive number $K_{m}$ for a small $\theta_{1}$ such that

$$
\left\|\Delta d^{m} f\right\| \leq K_{m} v_{m}(\varepsilon) \text { implies }\left\|\Delta d^{m} \psi\right\| \leq K_{m} v_{m}(\varepsilon)
$$

for $m=2,3, \cdots, l^{\prime}$, where $f \in \Gamma$ and $\Phi(f)=\psi$. Since $g_{0} \in \Gamma$ and $\left\|\Delta d^{m} g_{0}\right\| \leq$ $K_{m} v_{m}(\varepsilon)$ for $m=2,3, \cdots, l^{\prime}$, we have $\left\|\Delta d^{m} g_{k}\right\| \leq K_{m} v_{m}(\varepsilon)$ for $k=1,2, \cdots$. That is, $\left\{d^{m} g_{0}, d^{m} g_{1}, \cdots\right\}$ is equi-continuous. Thus, $g$ is of class $C^{\prime \prime}$ and $g \in \Gamma$.

This completes the proof of Theorem 1 .

Proof of Corollary.

If $y=g(\tau, x)$ for $\tau \in T$ and $\|x\| \leq \delta$, then

$$
\|y\| \leq\|g(\tau, x)-g(\tau, 0)\| \leq \sigma_{1}\|x\| \leq \delta .
$$

It is trivial that

$$
y_{0}=g\left(\tau_{0}, x_{0}\right) \text { implies } y_{1}=g\left(\tau_{1}, x_{1}\right)
$$

By (1.22) and (1.23), we see that $y_{0}=g\left(\tau_{0}, x_{0}\right)$ implies $\left(\tau_{m}, x_{m}, y_{m}\right) \in T \times B_{1}(\delta)$ $\times B_{2}(\delta)$ and $y_{m}=g\left(\tau_{m}, x_{m}\right)$ for any $m$. Suppose $y_{0}=g\left(\tau_{0}, x_{0}\right)$. From the equations $x_{1}=L_{1} x_{0}+\varphi_{1}\left(\tau_{0}, x_{0}, y_{0}\right)$ and $y_{1}=g\left(\tau_{1}, x_{1}\right)$, we have

$$
\begin{aligned}
\left\|y_{1}\right\| & \leq\left\|x_{1}\right\| \leq a\left\|x_{0}\right\|+\left\|\varphi_{1}\left(\tau,{ }_{0} x_{0}, y_{0}\right)-\varphi_{1}\left(\tau_{0}, x_{0}, 0\right)\right\| \\
& +\left\|\varphi_{1}\left(\tau_{0}, x_{0}, 0\right)-\varphi_{1}\left(\tau_{0}, 0,0\right)\right\| \leq a\left\|x_{0}\right\|+\theta_{1}\left\|y_{0}\right\|+\theta_{1}\left\|x_{0}\right\| \leq\left(a+2 \theta_{1}\right)\left\|x_{0}\right\| .
\end{aligned}
$$

Therefore, $\left\|y_{m}\right\| \leq\left\|x_{m}\right\| \leq\left(a+2 \theta_{1}\right)^{m}\left\|x_{0}\right\|$. Let $a+2 \theta_{1}<1$. Then we prove the first assertion of the corollary.

Ino rder to see that $y_{0} \neq g\left(\tau_{0}, x_{0}\right)$ implies $y_{1} \neq g\left(\tau_{1}, x_{1}\right)$, it is enough to 
consider the case of $\left\|x_{1}\right\| \leq \delta$ and $\left\|y_{1}\right\| \leq r_{0}$. From the equations

$$
\begin{aligned}
\tau_{1} & =\xi\left(\tau_{0}, x_{0}, y_{0}\right), \\
x_{1} & =L_{1} x_{0}+\varphi_{1}\left(\tau_{0}, x_{0}, y_{0}\right), \\
y_{1} & =L_{2} y_{0}+\varphi_{2}\left(\tau_{0}, x_{0}, y_{0}\right), \\
g\left(\tau_{0}, x_{0}\right)= & L_{2}^{-1}\left[g\left(\xi\left(\tau_{0}, x_{0}, g\left(\tau_{0}, x_{0}\right)\right), L_{1} x_{0}+\varphi_{1}\left(\tau_{0}, x_{0}, g\left(\tau_{0}, x_{0}\right)\right)\right)\right. \\
& \left.-\varphi_{2}\left(\tau_{0}, x_{0}, g\left(\tau_{0}, x_{0}\right)\right)\right],
\end{aligned}
$$

we have the following inequalities:

$$
\begin{aligned}
& \left\|y_{0}-g\left(\tau_{0}, x_{0}\right)\right\| \\
\leq & \left\|L_{2}^{-1}\right\| \| y_{1}-\varphi_{2}\left(\tau_{0}, x_{0}, y_{0}\right)-g\left(\xi\left(\tau_{0}, x_{0}, g\left(\tau_{0}, x_{0}\right)\right), L_{1} x_{0}+\varphi_{1}\left(\tau_{0}, x_{0}, g\left(\tau_{0}, x_{0}\right)\right)\right) \\
& +\varphi_{2}\left(\tau_{0}, x_{0}, g\left(\tau_{0}, x_{0}\right)\right) \| \\
\leq & \frac{1}{b}\left[\left\|y_{1}-g\left(\tau_{1}, x_{1}\right)\right\|+\| g\left(\tau_{1}, L_{1} x_{0}+\varphi_{1}\left(\tau_{0}, x_{0}, y_{0}\right)\right)-g\left(\tau_{1}, L_{1} x_{0}\right.\right. \\
& \left.+\varphi_{1}\left(\tau_{0}, x_{0}, g\left(\tau_{0}, x_{0}\right)\right)\right)\|+\| g\left(\xi\left(\tau_{0}, x_{0}, y_{0}\right), L_{1} x_{0}+\varphi_{1}\left(\tau_{0}, x_{0}, g\left(\tau_{0}, x_{0}\right)\right)\right) \\
& -g\left(\xi\left(\tau_{0}, x_{0}, g\left(\tau_{0}, x_{0}\right)\right), L_{1} x_{0}+\varphi_{1}\left(\tau_{0}, x_{0}, g\left(\tau_{0}, x_{0}\right)\right)\right)\|+\| \varphi_{2}\left(\tau_{0}, x_{0}, g\left(\tau_{0}, x_{0}\right)\right) \\
& \left.-\varphi_{2}\left(\tau_{0}, x_{0}, y_{0}\right) \|\right] \\
\leq & \frac{1}{b}\left[\left\|y_{1}-g\left(\tau_{1}, x_{1}\right)\right\|+\sigma_{1} \theta_{1}\left\|y_{0}-g\left(\tau_{0}, x_{0}\right)\right\|+\sigma_{1} c\left\|y_{0}-g\left(\tau_{0}, x_{0}\right)\right\|\right. \\
& \left.+\theta_{1}\left\|y_{0}-g\left(\tau_{0}, x_{0}\right)\right\|\right] .
\end{aligned}
$$

Then $\left(b-\sigma_{1} \theta_{1}-\sigma_{1} c-\theta_{1}\right)\left\|y_{0}-g\left(\tau_{0}, x_{0}\right)\right\| \leq\left\|y_{1}-g\left(\tau_{1}, x_{1}\right)\right\|$. Put $d=b-\sigma_{1} \theta_{1}-$ $\sigma_{1} c-\theta_{1}$. Then $d=1+b(1-k)>1$ since $k<1$. If $\left\|x_{k}\right\| \leq \sigma$ and $\left\|y_{k}\right\| \leq r_{0}$ for $k=1,2, \cdots, m$, then $d^{m}\left\|y_{0}-g\left(\tau_{0}, x_{0}\right)\right\| \leq\left\|y_{m}-g\left(\tau_{m}, x_{m}\right)\right\|$. Next,

$$
\begin{aligned}
\left\|\left(x_{m}, y_{m}\right)\right\| & \geq\left\|y_{m}\right\| \geq\left\|y_{m}-g\left(\tau_{m}, x_{m}\right)\right\|-\left\|g\left(\tau_{m}, x_{m}\right)\right\| \\
& \geq\left\|y_{m}-g\left(\tau_{m}, x_{m}\right)\right\|-\left\|x_{m}\right\| \\
& \geq\left\|y_{m}-g\left(\tau_{m}, x_{m}\right)\right\|-\left\|\left(x_{m}, y_{m}\right)\right\| .
\end{aligned}
$$

Therefore, $\left\|\left(x_{m}, y_{m}\right)\right\| \geq \frac{1}{2}\left\|y_{m}-g\left(\tau_{m}, x_{m}\right)\right\| \geq \frac{1}{2} d^{m}\left\|y_{0}-g\left(\tau_{0}, x_{0}\right)\right\|$. Thus, the second assertion of the corollary is proved.

\section{§2. The stable manifold of a Flow.}

Let $T, E_{i}$ and $B_{i}\left(r_{0}\right)$ for $i=1,2$ be the same as in $\S 1$. Let $\psi_{i}: T \times$ $B_{1}\left(r_{0}\right) \times B_{2}\left(r_{0}\right) \rightarrow E_{i}$ be of class $C^{l}$ such that $\psi_{i}(\tau, 0,0)=0$ and $d_{(2,3)} \psi_{i}(\tau, 0,0)=0$ for $i=1,2$. Let $P_{i}$ be a $k_{\imath} \times k_{i}$ matrix for $i=1,2$. Assume that the real parts of the eigen values of $P_{1}$ (resp. $P_{2}$ ) are negative (resp. positive). If 
we put $\left\|e^{P_{1}}\right\|=a$ and $\left\|e^{-P_{2}}\right\|=\frac{1}{b}$, then $a<1<b$. We shall denote by $T_{\tau}$ the tangent space at a point $\tau$ of $T$. Let $\xi: T \times B_{1}\left(r_{0}\right) \times B_{2}\left(r_{0}\right) \rightarrow \underset{\tau \in T}{\cup} T_{\tau}$ be of class $C^{l}$ such that $\xi(\tau, x, y) \in T_{\tau}$. Using a local coordinate neighborhood in $T$, we can express $\xi$ as a map from $E^{q} \times B_{1}\left(r_{0}\right) \times B_{2}\left(r_{0}\right)$ to $E^{q}$, where $E^{q}$ is a Euclidean space. We suppose $d \xi(\tau, 0,0)=0$ for any $\tau \in T$.

Let $f$ be a function of class $C^{l}$ from $E_{1} \times E_{2}$ to the set of all real numbers such that

$$
\begin{gathered}
0 \leq f(x, y) \leq 1 \text { for any }(x, y) \in E_{1} \times E_{2}, \\
f(x, y)=1 \text { for }\|x\| \leq \frac{r_{0}}{3} \text { and }\|y\| \leq \frac{r_{0}}{3}, \\
f(x, y)=0 \text { for }\|x\| \geq \frac{2}{3} r_{0} \text { or }\|y\| \geq \frac{2}{3} r_{0} .
\end{gathered}
$$

We consider a vector field $X$ on $T \times E_{1} \times E_{2}$ such that

(2.4) $X=\left(f(x, y) \xi(\tau, x, y), P_{1} x+f(x, y) \psi_{1}(\tau, x, y), P_{2} y+f(x, y) \psi_{2}(\tau, x, y)\right)$

on $T \times B_{1}\left(r_{0}\right) \times B_{2}\left(r_{0}\right)$,

$$
X=\left(0, P_{1} x, P_{2} y\right) \text { on }\|x\| \geq \frac{2}{3} r_{0} \text { or }\|y\| \geq \frac{2}{3} r_{0} .
$$

This vector field $X$ is an extension of a vector field $\left(\xi(\tau, x, y), P_{1} x+\psi_{1}(\tau, x, y)\right.$, $\left.P_{2} y+\phi_{2}(\tau, x, y)\right)$ on $T \times B_{1}\left(\frac{r_{0}}{3}\right) \times B_{2}\left(\frac{r_{0}}{3}\right)$. Let $\left(\Xi(t, \tau, x, y), \Phi_{1}(t, \tau, x, y)\right.$, $\left.\Phi_{2}(t, \tau, x, y)\right)$ be an integral curve for $X$ with initial value $(\tau, x, y)$ for $-\alpha<t<\beta \quad(\alpha, \beta>0)$, where the interval $(-\alpha, \beta)$ is the maximal interval for the existence of the solution for $X$. If $\beta<+\infty$, then a part of this integral curve is out of $T \times B_{1}\left(\frac{2}{3} r_{0}\right) \times B_{2}\left(\frac{2}{3} r_{0}\right)$ by p. 65 of Lang [2]. Since $X$ equals to $\left(0, P_{1} x, P_{2} y\right)$ in the exterior of $T \times B_{1}\left(\frac{2}{3} r_{0}\right) \times B_{2}\left(\frac{2}{3} r_{0}\right)$, we can extend this integral curve. This contradicts the assumption $\beta<+\infty$. Thus, $\beta=+\infty$. And also $\alpha=+\infty$. Therefore, the vector field $X$ is complete. Let $F^{t}$ be the one-parameter group generated by $X$. Then $F^{t}(\tau, x, y)=$ $\left(\boldsymbol{\Xi}(t, \tau, x, y), \Phi_{1}(t, \tau, x, y), \Phi_{2}(t, \tau, x, y)\right)$ for any $t$ and any $(\tau, x, y) \in T \times E_{1} \times$ $E_{2}$. Put $\Phi_{1}(t, \tau, x, y)=e^{P_{1} t} x+\varphi_{1}(t, \tau, x, y)$ and $\Phi_{2}(t, \tau, x, y)=e^{P_{2} t} y+\varphi_{2}(t, \tau, x, y)$. From the initial value condition $(\tau, x, y)$, we have $\Xi(0, \tau, x, y)=\tau$ and $\varphi_{i}(0, \tau, x, y)=0$ for $i=1,2$. Also we have $\varphi_{i}(t, \tau, 0,0)=0$ since the solution for $X$ with initial value $(\tau, 0,0)$ belongs to $T \times 0 \times 0$. Denote by $Y(t, \tau, x, y)$ the Jacobian of $\left(\xi, \Phi_{1}, \Phi_{2}\right)$ with respect to $(\tau, x, y)$ and denote by $A(\tau, x, y)$ 
the Jacobian of $\left(\xi, P_{1}+\phi_{1}, P_{2}+\phi_{2}\right)$ with respect to $(\tau, x, y)$ for $\|x\|,\|y\| \leq r_{0}$. Put $B=\left(\begin{array}{ccc}0 & 0 & 0 \\ 0 & P_{1} & 0 \\ 0 & 0 & P_{2}\end{array}\right)$. By solving the following variation equations with respect to the initial value $(\tau, 0,0)$,

$$
\begin{array}{r}
\frac{d}{d t} Y(t, \tau, 0,0)=A(\Xi(t, \tau, 0,0), 0,0) Y(t, \tau, 0,0), \\
Y(0, \tau, 0,0)=E \quad(E \text { is a unit matrix }),
\end{array}
$$

we have $d_{(2,3)} \varphi_{i}(t, \tau, 0,0)=0$ for $i=1,2$ and a sufficiently small $t$. Using the compactness of $T$ and the property of one-parameter group $F^{t}$, we have $d_{(2,3)} \varphi_{i}(t, \tau, 0,0)=0$ for any $t$ and $\tau \in T$. Moreover, we have $\Xi(t, \tau, x, y) \equiv$ constant and $\varphi_{i}(t, \tau, x, y) \equiv 0$ for $\|x\| \geq s_{0}$ or $\|y\| \geq s_{0}$, where $s_{0}$ is determined by $r_{0}$ and $s_{0} \rightarrow 0$ as $r_{0} \rightarrow 0$. We note that $\left\|d \varphi_{i}(t, \tau, x, y)\right\| \rightarrow 0$ as $s_{0} \rightarrow 0$ for $0 \leq t \leq 1$.

Put $M_{1}=\max \left\{\|A(\tau, x, y)\| \|(\tau, x, y) \in T \times B_{1}\left(r_{0}\right) \times B_{2}\left(r_{0}\right)\right\}$ and $M_{2}=\max _{0 \leq t \leq 1}\left\|e^{B t}\right\|$. Let $\varepsilon$ be a small positive number such that $c \equiv 1+3 \varepsilon M_{2} \frac{e^{M_{1}}-1}{M_{1}}<b$. For $M_{1}=0, \frac{e^{M_{1}}-1}{M_{1}}$ will be replaced by 1 . From the properties of $\hat{\xi}, \phi_{1}$ and $\psi_{2}$, we can choose a small positive number $r_{1}\left(\leq \frac{r_{0}}{3}\right)$ such that $\left\|d \psi_{1}(\tau, x, y)\right\|$ $\leq \varepsilon,\left\|d \psi_{2}(\tau, x, y)\right\| \leq \varepsilon$ and $\|d \xi(\tau, x, y)\| \leq \varepsilon$ for $\tau \in T,\|x\| \leq r_{1}$ and $\|y\| \leq r_{1}$. Now we consider the following variation equations with respect to the initial value $(\tau, x, y)$.

$$
\begin{gathered}
\frac{d}{d t} Y(t, \tau, x, y)=A\left(F^{t}(\tau, x, y)\right) Y(t, \tau, x, y), \\
Y(0, \tau, x, y)=E .
\end{gathered}
$$

From the properties of $\Phi_{1}$ and $\Phi_{2}$, we can choose a small positive number $r_{2}\left(\leq r_{1}\right)$ such that $\left\|\Phi_{i}(t, \tau, x, y)\right\| \leq r_{1}$ for $\|x\| \leq r_{2}$ and $\|y\| \leq r_{2}$. Since

$$
\begin{aligned}
& \left\|B e^{B t}-A\left(F^{t}(\tau, x, y)\right) e^{B t}\right\| \\
& \leq\left\|\left.\begin{array}{ccc}
\frac{\partial}{\partial \tau} \xi & \frac{\partial}{\partial x} \xi & \frac{\partial}{\partial y} \xi \\
\frac{\partial}{\partial \tau} \psi_{1} & \frac{\partial}{\partial x} \psi_{1} & \frac{\partial}{\partial y} \phi_{1} \\
\frac{\partial}{\partial \tau} \psi_{2} & \frac{\partial}{\partial x} \psi_{2} & \frac{\partial}{\partial y} \psi_{2}
\end{array}\right|_{F\left({ }^{t}(\tau, x, y)\right)}\right\|\|\| e^{B t} \|
\end{aligned}
$$


$\leq 3 \varepsilon M_{2}$ for $0 \leq t \leq 1,\|x\| \leq r_{2}$ and $\|y\| \leq r_{2}$,

$e^{B t}$ is an $3 \varepsilon M_{2}$-approximate solution of (2.8). By p. 56 of Lang [2], we have the following inequality,

$$
\left\|Y(t, \tau, x, y)-e^{B t}\right\| \leq 3 \varepsilon M_{2} \frac{e^{M_{1}|t|}-1}{M_{1}}
$$

for $0 \leq t \leq 1,\|x\| \leq r_{2}$ and $\|y\| \leq r_{2}$. For $t=1$, we have

$$
\left\|Y(1, \tau, x, y)-e^{B}\right\| \leq 3 \varepsilon M_{2} \frac{e^{M_{1}}-1}{M_{1}} .
$$

That is, $\left\|\frac{\partial}{\partial \tau} \Xi(1, \tau, x, y)-E\right\| \leq 3 \varepsilon M_{2} \frac{e^{M_{1}}-1}{M_{1}}$,

$$
\begin{aligned}
& \left\|\frac{\partial}{\partial x} \Xi(1, \tau, x, y)\right\| \leq 3 \varepsilon M_{2} \frac{e^{M_{1}}-1}{M_{1}} \text { and } \\
& \left\|\frac{\partial}{\partial y} \Xi(1, \tau, x, y)\right\| \leq 3 \varepsilon M_{2} \frac{e^{M_{1}}-1}{M_{1}} \text { for }\|x\|,\|y\| \leq r_{2} .
\end{aligned}
$$

Therefore, we have $\|d \Xi(1, \tau, x, y)\| \leq 1+3 \varepsilon M_{2} \frac{e^{M_{1}}-1}{M_{1}}=c<b$ for $\|x\|,\|y\| \leq r_{2}$. The map $F^{1}(\tau, x, y)=\left(\Xi(1, \tau, x, y), e^{P_{1} x}+\varphi_{1}(1, \tau, x, y), e^{P_{2}} y+\varphi_{2}(1, \tau, x, y)\right)$ : $T \times B_{1}\left(r_{2}\right) \times B_{2}\left(r_{2}\right) \rightarrow T \times E_{1} \times E_{2}$ satisfies the assumption of Theorem 1. Therefore, there exist $\delta>0$ and a $C^{1}$-map $g$ defined on $T \times B_{1}(\delta) \times B_{2}(\delta)$ to $E_{2}$ such that

$$
\begin{gathered}
g(\tau, 0)=0, d_{2} g(\tau, 0)=0 \text { for any } \tau \in T, \\
g\left(\Xi(1, \tau, x, g(\tau, x)), e^{P_{1} x}+\varphi_{1}(1, \tau, x, g(\tau, x))\right. \\
=e^{P_{2}} g(\tau, x)+\varphi_{2}(1, \tau, x, g(\tau, x)) .
\end{gathered}
$$

We note that $g$ is a $C^{l^{\prime}}$-map if $c, c{ }^{2}, \cdots, c^{l^{\prime}}<b \quad\left(l^{\prime} \leq l\right)$. Let $V$ be the local stable manifold of a diffeomorphism $F^{1}$.

Theorem 2. Let $\left(\tau_{t}, x_{t}, y_{t}\right)=F^{t}\left(\tau_{0}, x_{0}, y_{0}\right)$ for $\left(\tau_{0}, x_{0}, y_{0}\right) \in T \times E_{1} \times E_{2}$.

If $\left(\tau_{0}, x_{0}, y_{0}\right) \in V$, then there exists $n_{0}>0$ such that $\left(\tau_{t}, x_{t}, y_{t}\right) \in V$ for $t \geq n_{0}$ and $\left\|\left(x_{t}, y_{t}\right)\right\|=O\left(\left(a+2 \theta_{0}\right)^{t}\right)$ as $t \rightarrow \infty$, where $\theta_{0}$ is a sufficiently small positive number.

If $\left(\tau_{0}, x_{0}, y_{0}\right) \notin V$ and $\left\|x_{s}\right\|,\left\|y_{s}\right\| \leq \delta$ for $0 \leq s \leq t+n_{0}+1$, then $\left(\tau_{s}, x_{s}, y_{s}\right) \notin V$ and $\left\|\left(x_{s}, y_{s}\right)\right\| \geq K d^{s}$ for $s \leq t$, where $K$ is a positive constant and $d>1$.

Proof. We shall first verify that if $n \leq t \leq n+1$, then there exist positive constants $c_{1}, c_{2}$ independent of $n$ such that 


$$
c_{1}\left\|\left(x_{n}, y_{n}\right)\right\| \leq\left\|\left(x_{t}, y_{t}\right)\right\| \leq c_{2}\left\|\left(x_{n}, y_{n}\right)\right\| .
$$

In order to see this, note that $F^{t}=F^{t-n} \circ F^{n}$, that is,

$$
\begin{aligned}
& x_{t}=e^{P_{1}(t-n)} x_{n}+\varphi_{1}\left(t-n, \tau_{n}, x_{n}, y_{n}\right), \\
& y_{t}=e^{P_{2}(t-n)} y_{n}+\varphi_{2}\left(t-n, \tau_{n}, x_{n}, y_{n}\right) .
\end{aligned}
$$

From this equations, we have the following inequalities:

$$
\begin{aligned}
& \left\|x_{t}-e^{P_{1}(t-n)} x_{n}\right\| \leq \eta\left\|\left(x_{n}, y_{n}\right)\right\|, \\
& \left\|y_{t}-e^{P_{2}(t-n)} y_{n}\right\| \leq \eta\left\|\left(x_{n}, y_{n}\right)\right\|,
\end{aligned}
$$

where $\eta$ is a small positive number under a small $r_{0}$. Hence, we have $\left\|x_{t}\right\| \leq \eta\left\|\left(x_{n}, y_{n}\right)\right\|+\left\|e^{P_{1}(t-n)}\right\|\left\|x_{n}\right\|$. On the other hand,

$$
\begin{aligned}
\left\|x_{t}\right\| & \geq\left\|e^{P_{1}(t-n)} x_{n}\right\|-\left\|x_{t}-e^{P_{1} 1^{(t-n)}} x_{n}\right\| \\
& \geq \frac{1}{\left\|e^{-P_{1}(t-n)}\right\|}\left\|x_{n}\right\|-\eta\left\|\left(x_{n}, y_{n}\right)\right\| .
\end{aligned}
$$

For $y_{t}$, we have similar inequalities. Thus, we have (2.12).

There exists a natural number $n_{0}$ such that $c_{2}\left(a+2 \theta_{0}\right)^{n} \leq 1$ for $n \geq n_{0}$, where $\theta_{0}<\frac{1-a}{2}$. If $\left(\tau_{0}, x_{0}, y_{0}\right) \in V$, then $\left(\tau_{n}, x_{n}, y_{n}\right) \in V$ and $\left\|\left(x_{n}, y_{n}\right)\right\| \leq$ $\left(a+2 \theta_{0}\right)^{n}\left\|\left(x_{0}, y_{0}\right)\right\|$ for any $n$ from Corollary of Theorem 1. For $n_{0} \leq n \leq t \leq$ $n+1, \quad\left\|\left(x_{t}, y_{t}\right)\right\| \leq c_{2}\left\|\left(x_{n}, y_{n}\right)\right\| \leq c_{2}\left(a+2 \theta_{0}\right)^{n}\left\|\left(x_{0}, y_{0}\right)\right\| \leq \delta$. Therefore, we have $\left\|\left(x_{t}, y_{t}\right)\right\| \leq \delta$ for $t \geq n_{0}$ and $\lim _{t \rightarrow \infty}\left\|\left(x_{t}, y_{t}\right)\right\|=0$. Suppose that there exists $t_{0}$ such that $\left(\tau_{t_{0}}, x_{t_{0}}, y_{t_{0}}\right) \notin V$ and $t_{0} \geq n_{0}$. By Corollary of Theorem 1,

$$
\left\|\left(x_{t_{0}+k}, y_{t_{0}+k}\right)\right\| \geq \frac{1}{2} d^{k}\left\|\left(x_{t_{0}}, y_{t_{0}}\right)\right\| \text { for any } k \text {. }
$$

This is a contradiction. Therefore, we prove the first assertion of Theorem 2.

If $\left(\tau_{0}, x_{0}, y_{0}\right) \notin V$ and $\left\|x_{s}\right\|,\left\|y_{s}\right\| \leq \delta$ for $0 \leq s \leq t+n_{0}+1$, then we have $\left(\tau_{n}, x_{n}, y_{n}\right) \notin V$ and $\left\|\left(x_{n}, y_{n}\right)\right\| \geq \frac{1}{2} d^{n}\left\|\left(x_{0}, y_{0}\right)\right\|$ for $n \leq t+n_{0}+1$ by Corollary of Theorem 1. Suppose that there exists $t_{1}(\leq t)$ such that $\left(\tau_{t_{1}}, x_{t_{1}}, y_{t_{1}}\right) \in V$. By the above argument, we have $\left(\tau_{m}, x_{m}, y_{m}\right) \in V$ for $t_{1}+t_{0} \leq m \leq t+t_{0}+1$. This is a contradiction. The last inequality is trivial by (2.12).

By Theorem 2, we have

$$
V=\left\{\left(\tau_{0}, x_{0}, y_{0}\right) \in T \times B_{1}(\delta) \times B_{2}(\delta) \mid \lim _{t \rightarrow \infty} F^{t}\left(\tau_{0}, x_{0}, y_{0}\right) \in T \times 0 \times 0\right\} .
$$

We call this $V$ the local stable manifold of a flow $F^{t}$. If we put $W=$ 
$\cup F^{-t}(V)$, then $W$ is a $C^{1}$-manifold. We call this $W$ the stable manifold of a vector field $X$. The stable manifold $W$ is characterized as the set of $\left(\tau_{0}, x_{0}, y_{0}\right) \in T \times E_{1} \times E_{2}$ such that $\lim _{t \rightarrow \infty} F^{t}\left(\tau_{0}, x_{0}, y_{0}\right) \in T \times 0 \times 0$. Similarly, we can define the unstable manifolds of a vector field $X$.

\section{REFERENCES}

[1] N. Fenichel, Linearization of maps and flows near an invariant torus, Notices of the Amer. Math. Soc. 17 (1970), p. 406, 673-50.

[2] S. Lang, Introduction to differentiable manifolds, John Wiley and Sons, Inc., New York, 1962.

[3] P. Hartmann, Ordinary Differential Equations, John Wiley and Sons, Inc., New York, 1964.

[4] H. Rosenberg, A generalization of Morse-Smale inequalities, Bull. Amer. Math. Soc. 70 (1964), pp. 422-427.

[5] S. Smale, Morse inequalities for a dynamical system, Bull. Amer. Math. Soc. 66 (1960), pp. 43-49.

[6] S. Smale, Stable manifolds for differential equations and diffeomorphisms, Ann. Scuola Norm. Sup. Pisa (3) 17 (1963), pp. 97-116.

Mathematical Institute

Nagoya University 